TITLE:

Prescription of potentially inappropriate medications to elderly hemodialysis patients: prevalence and predictors( Dissertation_全文)

$\operatorname{AUTHOR}(S)$ :

Kondo, Naoya

CITATION:

Kondo, Naoya. Prescription of potentially inappropriate medications to elderly hemodialysis patients: prevalence and predictors. 京都大学, 2015, 博士(医学)

ISSUE DATE:

2015-01-23

URL:

https://doi.org/10.14989/doctor.k18676

RIGHT:

許諾条件により本文は2015/04/28に公開; This is a pre-copyedited, author-produced PDF of an article accepted for publication in Nephrology Dialysis Transplantation following peer review. The version of record "Prescription of potentially inappropriate medications to elderly hemodialysis patients: prevalence and predictors" is available online at: http://ndt.oxfordjournals.org/content/early/2014/04/26/ndt.gfu070.short. 


\section{Prescription of potentially inappropriate medications to elderly hemodialysis patients: prevalence and}

\section{predictors}

Naoya Kondo ${ }^{l}, M D$; Fumiaki Nakamura ${ }^{2}, M D, P h D$; Shin Yamazaki ${ }^{l}, P h D$; Yosuke Yamamoto ${ }^{l}, M D, P h D$;

Tadao Akizawa ${ }^{3}, M D, P h D$; Takashi Akiba 4 , MD, PhD; Akira Saito ${ }^{5}, M D, P h D ;$ Kiyoshi Kurokawa ${ }^{6}, M D$, PhD; Shunichi Fukuhara ${ }^{1,7,8}, M D, M S c, D M S c$

${ }^{1}$ Department of Healthcare Epidemiology, Kyoto University Graduate School of Medicine and Public Health, Kyoto, Japan

${ }^{2}$ Department of Public Health, The University of Tokyo Graduate School of Medicine, Tokyo, Japan

${ }^{3}$ Division of Nephrology, Department of Medicine, Showa University School of Medicine, Tokyo, Japan

${ }^{4}$ Department of Blood Purification and Internal Medicine, Kidney Center, Tokyo Women's Medical

University, Tokyo, Japan

${ }^{5}$ Division of Nephrology and Metabolism, Department of Medicine, Tokai University School of Medicine,

Kanagawa, Japan

${ }^{6}$ National Graduate Institute for Policy Studies, Tokyo, Japan

${ }^{7}$ Fukushima Medical University Center for Innovative Research in Clinical Evaluative Science, Fukushima,

Japan

${ }^{8}$ Institute for Health Outcomes and Process Evaluation Research, Japan 


\section{Corresponding author:}

Shunichi Fukuhara, MD, MSc, DMSc.

Department of Healthcare Epidemiology

Kyoto University Graduate School of Medicine and Public Health

Yoshida-Konoe-cho, Sakyo-ku 


\begin{abstract}
Background: In elderly hemodialysis patients, the risk of medication-related problems is particularly high. Thus, certain medications should generally not be prescribed to those patients. The Beers criteria for potentially inappropriate medications (PIMs) have been publicized. Still, with regard to elderly hemodialysis patients, the prevalence and risk factors for prescription of PIMs are unknown.
\end{abstract}

Methods: This was a cross-sectional study of data from the Japan Dialysis Outcomes and Practice Patterns Study (2002-2008). Patients were included if they were 65 years old or older and were currently receiving hemodialysis treatment at a hospital or clinic. We counted the number of patients who prescribed at least one PIM, as defined by the modified Beers criteria. We used multiple logistic regression analysis to determine which patient characteristics and facility characteristics were associated with prescription of PIMs.

Results: Data from 1367 elderly patients were analyzed. More than half of the patients (57\%) had been prescribed a PIM. The three most frequently prescribed PIMs were H2 blockers (33\%), anti-platelet agents (19\%), and alpha-blockers (13\%). PIM prescriptions were less likely at facilities that conducted multidisciplinary rounds (adjusted odds ratio [AOR]: 0.67 [95\% confidence interval $\{\mathrm{CI}\}: 0.48-0.93]$ ) and at teaching hospitals (AOR: 0.59 [95\% CI: 0.39-0.90]). PIM prescriptions are more likely if more than one physician has clearance to alter the hemodialysis regimen (AOR: 1.65 [95\% CI: 1.12-2.44]).

Conclusions: PIMs were prescribed to many elderly hemodialysis patients in Japan. Nephrologists should become more aware of PIMs. Multidisciplinary rounds could benefit patients by reducing the prescription of PIMs. 
Key words: DOPPS, potentially inappropriate medication, adverse drug events, hemodialysis, elderly patients

\section{Short summary:}

Studying elderly hemodialysis patients, we used existing criteria of potentially inappropriate medications (PIM), and we estimated both the frequency and the risk factors for prescription of PIM by data from the Japan DOPPS (Dialysis Outcomes and Practice Patterns Study). To the best of our knowledge, this is the first report of the frequency of prescriptions of PIM in hemodialysis patients. We found that PIM were prescribed more frequently in elderly hemodialysis patients than was previously reported in other elderly patients in Japan. Our results give a message to clinicians to pay more attention to how we manage this issue in elderly hemodialysis patients. 


\section{Introduction}

Issues associated with medication administration remain a major health care concern, particularly among elderly patients. A 2005 study in the United States found that adverse drug events (ADEs) occurred relatively frequently among ambulatory patients, with $27.6 \%$ found to be avoidable.[1] Hemodialysis patients are considered to be at higher risk for medication-related problems than the general population for several reasons, including impaired drug clearance,[2] increased frequency of polypharmacy, increased number of comorbidities, and increased proportion of receiving drugs that require therapeutic drug monitoring.[3] However, while previous studies have revealed that $98 \%$ of HD patients had at least one medication-related problem,[4] no efficient solutions have yet been proposed.

Identifying drugs carrying high risk of ADEs is one possible strategy for managing medication-related problems. In theory, reducing the likelihood of physicians prescribing such drugs consequently reduces the incidence of medication-related problems and ADEs.[5] These high-risk drugs are called "potentially inappropriate medication (PIM)" and are defined as "medication with no clear evidence-based indication, and which carry a substantially higher risk of adverse side-effects or are not cost-effective".[6] Several sets of criteria for PIMs have been developed specifically for use with elderly patients, with the Beers criteria the most commonly used in previous epidemiological studies.[6] Akazawa et al. reported that the frequency of prescribing PIM, as defined using a modified version of the Beers criteria reflecting regional clinical practice and available medications in Japan, was $43.6 \%$ among elderly patients in Japan.[7] However, this study population was made up of beneficiaries covered by the employees' health insurance system, which included healthier individuals than may be found in the general elderly population. As such, prescription patterns of 
PIM for patients with severe disease remain unclear.

Previous reports on the employees' health system have all involved relatively heterogeneous general elderly populations, failing to account for the fact that prescription patterns of PIMs may differ according to patients' comorbidities. A study surveying prescription patterns of these medications of elderly hemodialysis patients may therefore provide important information on the subject of the difference between healthy elderly patients and elderly patients with severe disease. Here, we attempted to determine the prevalence of and identify risk factors for prescribing PIMs in elderly hemodialysis patients. 


\section{Method}

Study design and data source

We obtained all data from phases II (from year 2002 to 2004) and III (from year 2005 to 2008) of the Dialysis Outcome Practice Pattern Study in Japan (J-DOPPS II and J-DOPPS III), which were large cohort studies involving detailed data from adult HD patients at more than 50 randomly selected dialysis facilities in Japan. DOPPS originally sought to determine dialysis practices which most contributed to improved mortality and hospitalization rates, health-related quality of life, and vascular access outcomes after adjusting for the effects of comorbid disease and other demographic confounding factors. The dialysis facilities included in DOPPS constitute a nationally representative sample. To ensure variation in practice patterns and outcomes, a stratified random sample of hemodialysis facilities was selected. DOPPS's methodology has been detailed previously, $[8,9]$ and all institutional review boards approved its conduct in each facility, as required.

Prescribed drug information and patients' demographic data were surveyed at study enrollment. This study was cross-sectional in design to examine the association between PIM and other factors.

\section{Study population}

To ensure a representative national sample, two-staged random sampling method was used in the J-DOPPS. After first randomly selecting our 50 hemodialysis facilities of focus, we then randomly selected patients at those facilities in each study phase. Inclusion criteria for the present study were an age of 65 years or older and currently receiving chronic hemodialysis treatment. Patients receiving transient dialysis were excluded. 


\section{Outcome measurement}

We used the modified Beers criteria for elderly Japanese populations to define PIMs,[7] identifying a total of 47 PIMs as selected by 9 expert panel members. The criteria consisted of either medications that should be always avoided or those which should be avoided only in particular situations. Participants receiving drug prescription classified as PIM only if prescribed long-term were additionally examined at one year after enrollment. Given that non-critical comorbidities such as insomnia were not recorded correctly in these studies, we defined insomniac patients as those who prescribed hypnotic agents and constipated patients as those who prescribed laxative agents. Medications that were to be avoided in patients with incontinence or urinary retention were excluded, as most hemodialysis patients are generally accepted to be anuric.

The primary outcome was prescribed at least one PIM as defined by the modified Beers criteria. Prevalence of PIM prescription was estimated. To evaluate changes over time in PIM prescription, we also compared the frequency of PIM prescription between J-DOPPS II and J-DOPPS III.

\section{Statistical analysis}

Differences in distributions of primary outcome within categorical valuables were compared using the $\chi^{2}$ test. Multiple logistic regression analysis was performed to determine patient characteristics associated with PIMs; the model included age, sex, vintage, number of co-morbidities, number of medications, dependency in activities in daily living (ADL; defined by using a wheelchair or similar aids), past history of depression, and living alone. Numbers of comorbidities and numbers of medications were divided into four groups based on 
variable quartile.

Given that facility characteristics were measured only in the J-DOPPS III cohort, we conducted sub-group analysis using only patients participating in J-DOPPS III to determine facility characteristic associated with prescription of PIMs. In this sub-group analysis, logistic regression analysis including the above patient characteristics and facility characteristics was performed. Number of hemodialysis stations was divided into dichotomous variables based on the median value. To estimate cluster effects of each facilities, two-stage random effect logistic regression analysis was also performed as sensitivity analysis.

Differences or associations with a two-sided $\mathrm{P}$ value of less than 0.05 were considered statistically significant. All analyses were performed using STATA version 11.2 (StataCorp LP, College Station, TX, USA). 


\section{Results}

A total of 1367 elderly patients were deemed eligible for this study (Figure 1), and their characteristics are shown in Table 1. Characteristics of patients were strikingly similar across both phases of J-DOPPS. Median age was 72 (range: $65-98$ ) years, and $33 \%$ of patients were found to have diabetes as the primary cause of end-stage renal disease. Only $1 \%$ of patients had a history of definite diagnosis of depression. Details of facility characteristics in the J-DOPPS III cohort are available in the online appendix. Teaching hospitals made up $26 \%$ of facilities examined, and almost half of all facilities conducted multidisciplinary rounds (57\%). At least two physicians decided on dialysis treatment in $67 \%$ of all facilities.

Figure 2 shows the distribution of the number of PIMs prescribed, revealing that most patients were prescribed one or two PIMs (52\%). Table 2 shows the frequency of prescription of PIM and detailed lists of medications among this population. More than half of patients were prescribed PIM-most often H2 blockers. A total of $38 \%$ of patients were prescribed famotidine at or exceeding $20 \mathrm{mg}$ daily, the usual dose for patients with normal kidney function. The second most frequently prescribed PIM was cardiovascular drugs (Table 2), primarily anti-platelet drugs such as ticlopidine (19\%) and alpha-blockers (13\%). As with patient characteristics, no remarkable differences in details of PIM were noted between the two J-DOPPS phases.

Table 3 describes proportions of PIM prescription stratified by patient characteristics (socio-demographic characteristics, health status, and number of all medications prescribed), while Figure 3 presents the results of multivariable logistic regression analysis. Longer vintage of hemodialysis was associated with increased proportion of inappropriate medication prescription $(<1$ year: $47 \%$, adjusted odds ratio [AOR] reference; 1 to 
4 years: $59 \%$, AOR 1.58 [95\% confidence interval $\{\mathrm{CI}\}: 1.15-2.17]$; more than 5 years: $59 \%$, AOR 1.77 [95\%

CI, 1.28-2.44]). An increase in proportion of prescribed any medication at all was also associated with prescription of PIMs. While number of comorbidities seemed to be associated with prescription of PIMs, the degree was not statistically significant in multivariable analysis. Of note, dependency in ADL (equal to or less than ADL when using a wheel chair ) was negatively associated with prescription of PIMs (AOR 0.56 [95\% CI, 0.39-0.82]). No significant association was noted between prescription of PIMs and age, sex, past history of depression, and living alone.

Table 4 presents the results of univariate analysis, and Figure 4 presents the results of multivariable analysis including facility factors in the J-DOPPS III cohort. Patients receiving hemodialysis at a facility with multidisciplinary rounds conducted (AOR 0.67, [95\% CI, 0.48-0.93]) and at teaching hospital (AOR 0.59, [95\% CI, 0.39-0.90]) were less frequently prescribed PIM. Patients receiving hemodialysis at a facility in which more than one physician had clearance to change the dialysis regimen had a higher risk of prescribed PIM (AOR 1.65, [95\% CI, 1.12-2.44]) than those receiving treatment as directed by one physician. Sensitivity analysis with two-staged random effect model showed no significant clustering at the facility level.

\section{Discussion}

The overall frequency of PIMs was 57\% among Japanese elderly hemodialysis patients, a finding similar between both J-DOPPS phases examined. The most frequently prescribed PIMs in our study were H2 blockers, anti-platelet agents, and alpha-blocker agents. We noted no remarkable differences in details of PIM between the two different phases of J-DOPPS. Patients on hemodialysis for a relatively long time, prescribed many medications, or treated by more than one physician were at greater risk of PIM prescription than those not 
meeting these criteria. In contrast, patients treated at teaching hospitals or hospitals conducting multidisciplinary rounds were at relatively low risk of PIM prescription. Taken together, these findings suggest that elderly hemodialysis patients were prescribed PIM more frequently than previously reported for the general elderly population.[7]

A previous study reported that $2.7 \%$ of general elderly patients were prescribed anti-platelet agents classified to a PIM,[7] compared with a proportion of $15 \%$ among elderly hemodialysis patients in the present study. Elderly hemodialysis patients were prescribed anti-platelet agents more often than non-hemodialysis patients given the increased incidence of vascular disease among elderly patients on hemodialysis. However, a previous study on antiplatelet therapy in vascular disease (ischemic stroke, coronary artery disease, and peripheral arterial disease) suggested that aspirin or clopidogrel should be used as first-line agents for the majority of the patients, as ticlopidine usage is limited by its life-threatening hematological adverse reactions including neutropenia, thrombotic thrombocytopenic purpura (TTP) and aplastic anemia. Although clopidogrel, a possible alternative to ticlopidine, was not available in Japan at study enrollment for either cohort, we considered that ticlopidine should be prescribed more carefully.

Frequent use of $\mathrm{H} 2$ blockers was deemed one of the reasons for the high frequency of prescription of PIMs in the present study. Previous report from worldwide DOPPS reveals that proton pump inhibitors (PPI) were used much less often than $\mathrm{H} 2$ blockers in Japan ( $0.8 \%$ vs. $31.6 \%$, respectively) and the frequency of prescription is very low compared to other country $(14.0 \%$ to $27.3 \%)[10]$. In Japan, the usual prescription of PPIs for gastric and duodenal ulcers is limited to 8 weeks. This limitation may affect the relatively high frequency of prescription of $\mathrm{H} 2$ blockers. $\mathrm{H} 2$ blockers are associated with mental status changes such as 
delirium and decline in cognitive function in elderly patients;[11] indeed, a previous study revealed that approximately $10 \%$ of adult end-stage renal disease patients with prescription of famotidine had shown mental status changes during over 7 years of follow-up.[12] While dose adjustment may decrease the frequency of $\mathrm{ADE}, 38 \%$ of patients were prescribed the usual dose of famotidine in the present study (20-40 mg daily).

While Akazawa et al. reported that $2.8 \%$ of general elderly patients were prescribed alpha-blockers classified to a PIM,[7] 12\% of elderly hemodialysis patients were prescribed these drugs in our study. Report from DOPPS reveals that vasodilators are less often prescribed for hemodialysis patients in Japan than other countries.[13] Another previous study reported that alpha-blockers approximately tripled the risk of falling,[14] and a previous observational study showed that hemodialysis patients with alpha-blocker prescriptions had slightly higher mortality independent of variables such as age, sex, race, years of end-stage renal disease, or prevalence of co-morbidities.[13] Other anti-hypertensive drugs such as angiotensin converting enzyme (ACE) inhibitors,[15] angiotensin receptor blockers (ARBs), and beta blockers,[13] which are all associated with reduced mortality risk in hemodialysis patients, should be prescribed instead of alpha-blockers.

Under the modified Beers criteria, the following analgesics are classified as PIMs: indomethacin, pentazocine, long-term use of full-dosage long half-life non-COX-selective NSAIDs, and general NSAIDs prescription in patients with gastric or duodenal ulcers. As such, analgesic drugs classified as PIMs were rarely prescribed in the present study.

Here, we identified several factors associated with prescription of PIM, with our findings for polypharmacy and dependency in ADL consistent with those of previous studies.[16] Our findings also suggested that longer 
vintage may be associated with time-dependent increase of cardiovascular or gastrointestinal complications; therefore, patients receiving hemodialysis for a relatively long period of time may have more chances to be prescribed PIMs than those with shorter vintage. In contrast, several variables (number of co-morbidities, age, sex, past history of depression, living alone) already known to be associated with prescription of PIMs[16] were not found to be statistically significantly associated in the present study. We believe that number of co-morbidities was not an important factor influencing PIMs in the present study because hemodialysis status involves serious comorbidities. Given that markedly few patients had a definite history of depression or were older than 85 years, we were unable to detect statistically significant differences in the frequency of PIMs among this population.

In multivariable analysis of the J-DOPPS III cohort, PIM prescription was found to be associated with several facility factors. Patients at teaching hospital may receive relatively high-quality treatment, thereby avoiding many medication-related problems. We further believe that multidisciplinary rounds were useful in facilitating sharing of information about ADEs between physicians and other health care providers, possibly resulting in avoiding prescription of PIM; this finding is consistent with previous studies, further underscoring the efficacy of a multidisciplinary team in avoiding PIM.[17] Having more than one dialysis physician make the decision to change hemodialysis regimen may increase the opportunity to be prescribed PIMs. Taken together, these findings suggest that prescription of PIM was affected by modifiable practice patterns.

Six major limitations to the present study warrant mention. First, because of the cross-sectional study design, causal inferences cannot be made. However, as mentioned above, variation in these facility factors (teaching hospital, multidisciplinary rounds conducted, or changing of the hemodialysis regimen by more than 
one physician) cannot logically be deemed to be due to prescription of PIM. We thought that these factors were the cause or preventive factors of PIM. Second, information regarding patient disease history was limited to 29 diseases, thereby reducing the frequency of PIMs to be taken into account in patients with certain disease such as hyponatremia. This limitation may have thereby led to underestimation of the frequencies of PIM. Third, as we used Beers criteria to identify PIMs, other medications that are at high risk for ADEs in hemodialysis patients may not be included. This limitation may also lead to underestimation of the frequencies of PIM. Fourth, some patients may have been redundantly selected for both the J-DOPPS II and J-DOPPS III through random sampling. However, we assume that the percentage of such patients is negligible. Fifth, the present study used data from Japanese hemodialysis patients; as such, global application of our findings will require further investigation. Sixth, association between PIMs and clinical outcome was unclear in elderly hemodialysis patients.[18] Further study will be needed to clearly determine the clinical impact of PIMs in this patient population.

In conclusion, the fact that PIMs were prescribed to more than half of the patients in this study underscores the importance of medication management in elderly people receiving hemodialysis. Nephrologists should be aware of the frequency and dangers of prescribing PIMs. In addition, we realize that for all hemodialysis facilities to implement all of the practices that are common in teaching hospitals might be impractical. Further research should be done on the practices that are common in teaching hospitals, to find out which among them most strongly inhibit the prescription of PIMs. We believe that, as a minimum, multidisciplinary rounds should be conducted at all facilities where they are possible. 


\section{Acknowledgments}

The DOPPS is supported by research grants from Amgen, Inc., and Kyowa Hakko Kirin Co., Ltd, without restriction on publication. T. Akizawa receives consulting fees from Chugai, Kyowa Kirin, Bayer, Astellas, REATA and Abbott, and grants/funds from Chugai Kyowa Kirin, Bayer, Astellas and Daiichi-Sankyo. S.

Fukuhara is an advisor on epidemiology studies for Kyowa Hakko Kirin and receives consulting fees from Kyowa Hakko Kirin. Other authors have nothing to declare. 


\section{References}

1. Gurwitz JH, Field TS, Judge J, et al. The incidence of adverse drug events in two large academic long-term care facilities. Am J Med 2005; 118: 251-258

2. Strand LM, Morley PC, Cipolle RJ, et al. Drug-related problems: their structure and function. DICP 1990; 24: 1093-1097

3. Koecheler JA, Abramowitz PW, Swim SE, et al. Indicators for the selection of ambulatory patients who warrant pharmacist monitoring. Am J Hosp Pharm 1989; 46: 729-732

4. Manley HJ, McClaran ML, Overbay DK, et al. Factors associated with medication-related problems in ambulatory hemodialysis patients. Am J Kidney Dis 2003; 41: 386-393

5. Fick D, Semla T, Beizer J, et al. American Geriatrics Society Updated Beers Criteria for Potentially Inappropriate Medication Use in Older Adults. J Am Geriatr Soc 2012; 60: 616-631

6. O'Mahony D, Gallagher PF. Inappropriate prescribing in the older population: need for new criteria. Age Ageing 2008; 37: 138-141

7. Akazawa M, Imai H, Igarashi A, et al. Potentially inappropriate medication use in elderly Japanese patients. Am J Geriatr Pharmacother 2010; 8: 146-160

8. Young EW, Goodkin DA, Mapes DL, et al. The Dialysis Outcomes and Practice Patterns Study (DOPPS): An international hemodialysis study. Kidney Int 2000; 57: S74-S81

9. Pisoni RL, Gillespie BW, Dickinson DM, et al. The Dialysis Outcomes and Practice Patterns Study (DOPPS): design, data elements, and methodology. Am J Kidney Dis 2004; 44: 7-15

10. Bailie GR, Mason NA, Elder SJ, et al. Large variations in prescriptions of gastrointestinal medications in hemodialysis patients on three continents: the Dialysis Outcomes and Practice Patterns Study (DOPPS). Hemodial Int 2006; 10: 180-188

11. Lai KV, Larson EB. Drug-Induced Cognition Disorders in the Elderly Incidence, Prevention and Management. Drug Saf 1999; 21: 101-122

12. Redmond AM, Pentapaty N, Weibel J, et al. Use of Famotidine in Adult Patients with End-Stage Renal Disease: Assessment of Dosing and Mental Status Changes. Am J Med Sci 2005; 330: 8-10

13. Lopes AA, Bragg-Gresham JL, Ramirez SP, et al. Prescription of antihypertensive agents to haemodialysis patients: time trends and associations with patient characteristics, country and survival in the DOPPS. Nephrol Dial Transplant 2009; 24: 2809-2816

14. Myers AH, Baker SP, Vannatta ML, et al. Risk factors associated with falls and injuries among elderly institutionalized persons. Am J Epidemiol 1991; 133: 1179-1190

15. Efrati S, Zaidenstein R, Dishy V, et al. ACE inhibitors and survival of hemodialysis patients. Am J Kidney Dis 2002; 40: 1023-1029

16. Fialova D, Topinkova E, Gambassi G, et al. Potentially inappropriate medication use among elderly home care patients in Europe. JAMA 2005; 293: 1348-1358

17. Cardone KE, Bacchus S, Assimon MM, et al. Medication-related problems in CKD. Adv Chronic Kidney Dis 2010; 17: 404-412

18. Jano E, Aparasu RR. Healthcare outcomes associated with Beers' criteria: A systematic review. Ann Pharmacother 2007; 41: 438-448 
Table 1. Patient Characteristics

\begin{tabular}{|c|c|c|c|}
\hline Characteristics & $\begin{array}{c}\text { J-DOPPS II } \\
(2002) \\
(\mathrm{N}=595) ; \%\end{array}$ & $\begin{array}{c}\text { J-DOPPS III } \\
(2005) \\
(\mathrm{N}=772) ; \% \\
\end{array}$ & $\begin{array}{c}\text { Overall } \\
(\mathrm{N}=1367) ; \%\end{array}$ \\
\hline \multicolumn{4}{|l|}{ Sex } \\
\hline Male & 57 & 60 & 59 \\
\hline \multicolumn{4}{|l|}{ Primary cause of ESRD } \\
\hline$D M$ & 32 & 34 & 33 \\
\hline \multicolumn{4}{|l|}{ Age (years) } \\
\hline $65-69$ & 31 & 36 & 34 \\
\hline $70-74$ & 34 & 27 & 30 \\
\hline $75-79$ & 20 & 21 & 20 \\
\hline $80-84$ & 9 & 10 & 10 \\
\hline$\geq 85$ & 5 & 6 & 6 \\
\hline \multicolumn{4}{|l|}{ Vintage (year) } \\
\hline$<1$ & 15 & 23 & 19 \\
\hline $1-4$ & 45 & 34 & 39 \\
\hline$\geq 5$ & 40 & 43 & 42 \\
\hline \multicolumn{4}{|l|}{ Number of comorbidities ${ }^{\text {a }}$} \\
\hline 0 & 6 & 5 & 5 \\
\hline $1-2$ & 18 & 37 & 29 \\
\hline $3-4$ & 42 & 30 & 35 \\
\hline$\geq 5$ & 33 & 28 & 30 \\
\hline \multicolumn{4}{|l|}{ Number of medications } \\
\hline$<6$ & 33 & 26 & 29 \\
\hline $6-7$ & 18 & 26 & 23 \\
\hline $8-9$ & 24 & 24 & 24 \\
\hline$\geq 10$ & 25 & 24 & 24 \\
\hline Past history of depression & 1 & 1 & 1 \\
\hline High dependency in ADL (e.g. using a wheelchair) & 13 & 12 & 12 \\
\hline Living alone & 9 & 10 & 10 \\
\hline $\begin{array}{l}\text { Receiving hemodialysis at large medical institution } \\
\text { (number of } \mathrm{HD} \text { stations } \geq \mathbf{3 0} \text { ) }\end{array}$ & $-\mathrm{b}$ & 43 & \\
\hline Receiving hemodialysis at teaching hospitals & $-b$ & 23 & \\
\hline $\begin{array}{l}\text { Receiving hemodialysis at medical institution with } \\
\text { multidisciplinary rounds conducted }\end{array}$ & $-b$ & 62 & \\
\hline $\begin{array}{l}\text { Receiving hemodialysis treatment by more than one } \\
\text { physician }\end{array}$ & $-{ }^{b}$ & 70 & \\
\hline
\end{tabular}


ESRD: end-stage renal disease, ADL: activities in daily living, HD: hemodialysis, DM: diabetes mellitus ${ }^{\mathrm{a}}$ Number of comorbidities: angina, myocardial infarction, arrhythmia, congestive heart failure, hypertension, hyperlipidemia, cerebrovascular disease, transient ischemic attacks, peripheral vascular disease, aortic aneurysm, claudication, past history of deep vein thrombosis, diabetes, chronic obstructive pulmonary disease, seizure disorder, dementia and other cognitive impairment, peripheral neuropathy, Parkinson's disease, depression, history of hip fractures, carpal tunnel syndrome, peptic ulcer disease, recent history gastrointestinal bleed, diabetic gastroparesis, ascites, viral hepatitis, recurrent cellulitis/skin infection/gangrene, cancer, and HIV/AIDS

${ }^{b}$ not measured 


\begin{tabular}{lccc}
\hline & J-DOPPS II & J-DOPPS III & \\
& $\mathbf{( 2 0 0 2 )}$ & $\mathbf{( 2 0 0 5 )}$ & Overall \\
& $\mathbf{N}=\mathbf{5 9 5}$ & $\mathbf{N}=\mathbf{7 7 2}$ & $\mathbf{N = 1 3 6 7}$ \\
\hline Any PIM, (\%) & 58 & 56 & 57 \\
\hline
\end{tabular}

\section{Cardiovascular drugs}

Antihypertensive drugs (\%)

14

12

13

Doxazosin (\%)

11

12

12

Prazosin Hydrochloride (\%)

$<1$

$<1$

$<1$

Methyldopa (\%)

2

2

2

Clonidine (\%)

1

1

Anti-arrhythmic drugs

5

3

4

Pilsicainide Hydrochloride (\%)

$<1$

$<1$

Disopyramide (\%)

2

1

Amiodarone Hydrochloride (\%)

$<1$

$<1$

Digoxin (\%)

$<1$

$<1$

Digitoxin (\%)

1

Propranolol Hydrochloride (\%)

1

Verapamil Hydrochloride (\%)

Antiplatelet drugs (\%)

19

19

19

Aspirin (\%)

1

6

4

Short-acting Dipyridamole (\%)

$<1$

2

1 
Ticlopidine Hydrochloride (\%)

19

14

16

Central nervous system depressant drugs

5

6

6

Etizolam (\%)

1

$<1$

$<1$

Benzodiazepine class (\%)

5

3

4

Diazepam (\%)

2

1

2

Ethyl Loflazepate (\%)

$<1$

$<1$

$<1$

Triazolam (\%)

$<1$

$<1$

$<1$

Flunitrazepam (\%)

2

1

2

Nitrazepam (\%)

$<1$

$<1$

$<1$

Brotizolam (\%)

$<1$

$<1$

$<1$

Alprazolam (\%)

$<1$

$<1$

$<1$

Amitriptyline Hydrochloride (\%)

$<1$

$<1$

$<1$

Milnacipran Hydrochloride (\%)

$<1$

$<1$

$<1$

$\mathbf{H}_{2}$ blockers (\%)

33

30

31

Famotidine (\%)

17

18

17

Ranitidine Hydrochloride (\%)

8

4

5

Cimetidine (\%)

3

2

3

Nizatidine (\%)

1

1

1

Lafutidine (\%)

2

4

3

Roxatidine Acetate Hydrochloride (\%)

2

1

1 
Miscellaneous drugs (\%)

Loxoprofen Sodium (\%)

Indomethacin (\%)

Zaltoprofen (\%)

Ampiroxicam (\%)

Diphenhydramine (\%)

Chlorpheniramine Maleate (\%)

Promethazine (\%)

Dihydroergotoxine Mesilate (\%)

Propantheline Bromide Chlorophyll

Combined Drug (\%)

Propiverine Hydrochloride (\%)
4

2

3

$<1$

$<1$

$<1$

$<1$

$<1$

$<1$

$<1$

0

$<1$

0

$<1$

$<1$

$<1$

0

$<1$

3

1

2

$<1$

$<1$

$<1$

$<1$

$<1$

$<1$

$<1$

$<1$

$<1$

$<1$

$<1$

$<1$ 
Table 3. Univariate analysis of potentially inappropriate medication and patient factors

\begin{tabular}{cc}
\hline Overall (J-DOPPS II \& III) \\
\cline { 2 - 3 } \\
Inappropriate medication & P value \\
\hline
\end{tabular}

Sex

Female

57

43

Male

57

43

0.930

\section{Primary cause of ESRD}

non-DM

$D M$

\section{Age (year)}

65-69

70-74

75-79

80-84

$\geq 85$

Vintage (year)

$<1$

$1-4$

$\geq 5$
54

62

57

54

57

62

55

47

59

59
46

38

0.006
43

46

43

38

45

0.603 


\section{Number of comorbidities}

$$
0
$$$$
1-2
$$$$
3-4
$$$$
\geq 5
$$

Number of medications

$$
<6
$$$$
\text { 6-7 }
$$$$
8-9
$$$$
\geq 10
$$

\section{Past history of depression}

No

Yes

\section{Low ADL}

(e.g. wheel-chair-bound)

$$
\text { No }
$$

Yes

\section{Living alone}

No

Yes
0.097

$$
66
$$$$
34
$$

25

$<0.001$
0.154

44

0.936 
ESRD: end-stage renal disease, DM: diabetes mellitus, ADL: activities in daily living 
Table 4. Univariate analysis of potentially inappropriate medication and facility factors in J-DOPPS III group $(\mathrm{N}=772)$

Inappropriate medication

P value

Yes $(\%) \quad$ No $(\%)$

Number of HD stations

$<30$

59

41

$\geq 30$

52

48

0.043

Teaching hospital

No

58

43

Yes

50

50

0.066

Multidisciplinary rounds conducted

No

58

43

Yes

50

50

0.085

Hemodialysis regimen able to be changed

by more than one physician

No

55

45

Yes

56

44

0.766

HD: hemodialysis 
Appendix table. Facility Characteristics

$$
\%(n=61
$$

Characteristics

facilities in total)

Number of HD stations $(\geq 30)$

Teaching hospital (yes)

26

Multidisciplinary rounds conducted (yes)

Recommendation for HD treatment made by more than one

physician (yes)

HD: hemodialysis 


\section{Figure legends}

Figure 1. Patient flow chart

HD: hemodialysis

Figure 2. Distribution of numbers of potentially inappropriate medication (PIM)

Figure 3. Multivariable analysis of potentially inappropriate medication and patient factors $(\mathrm{N}=1367)$

ADL: activities in daily living, DM: diabetes mellitus, ESRD: end-stage renal disease, OR: odds ratio, CI:

confidence interval

Figure 4. Multivariable analysis of potentially inappropriate medication and facility factors $(\mathrm{N}=772)$

HD: hemodialysis, OR: odds ratio, CI: confidence interval

The results presented in this paper have not been published previously. 
Figure 1

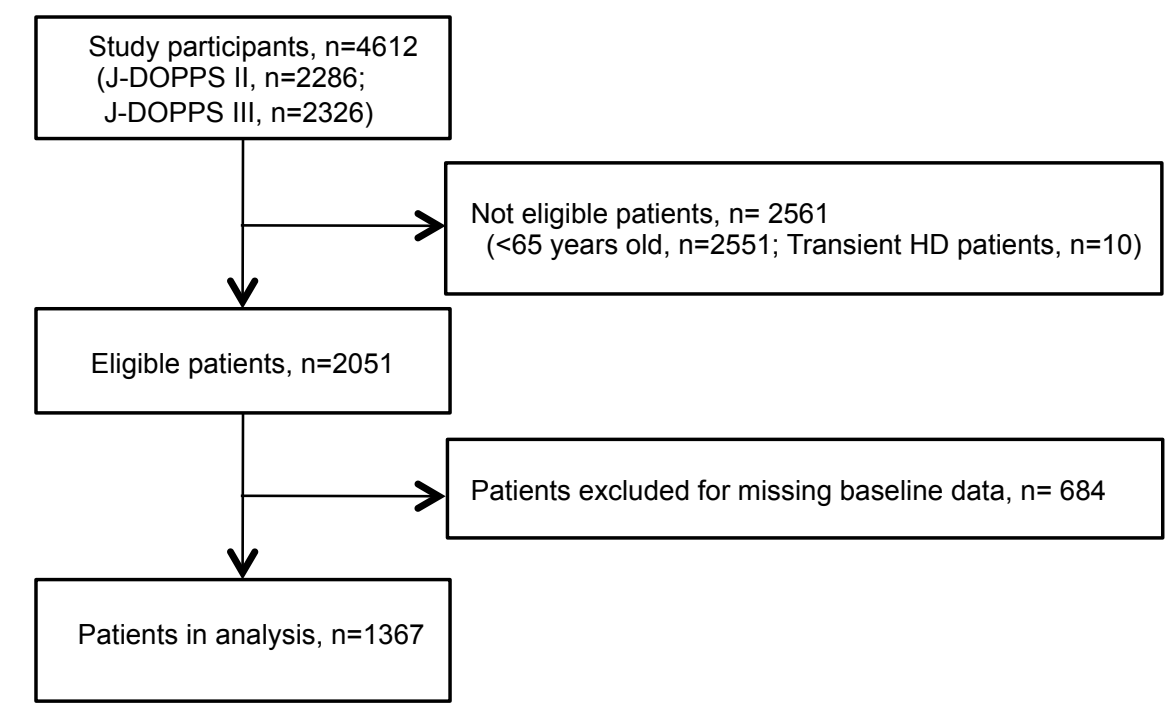


Figure 2

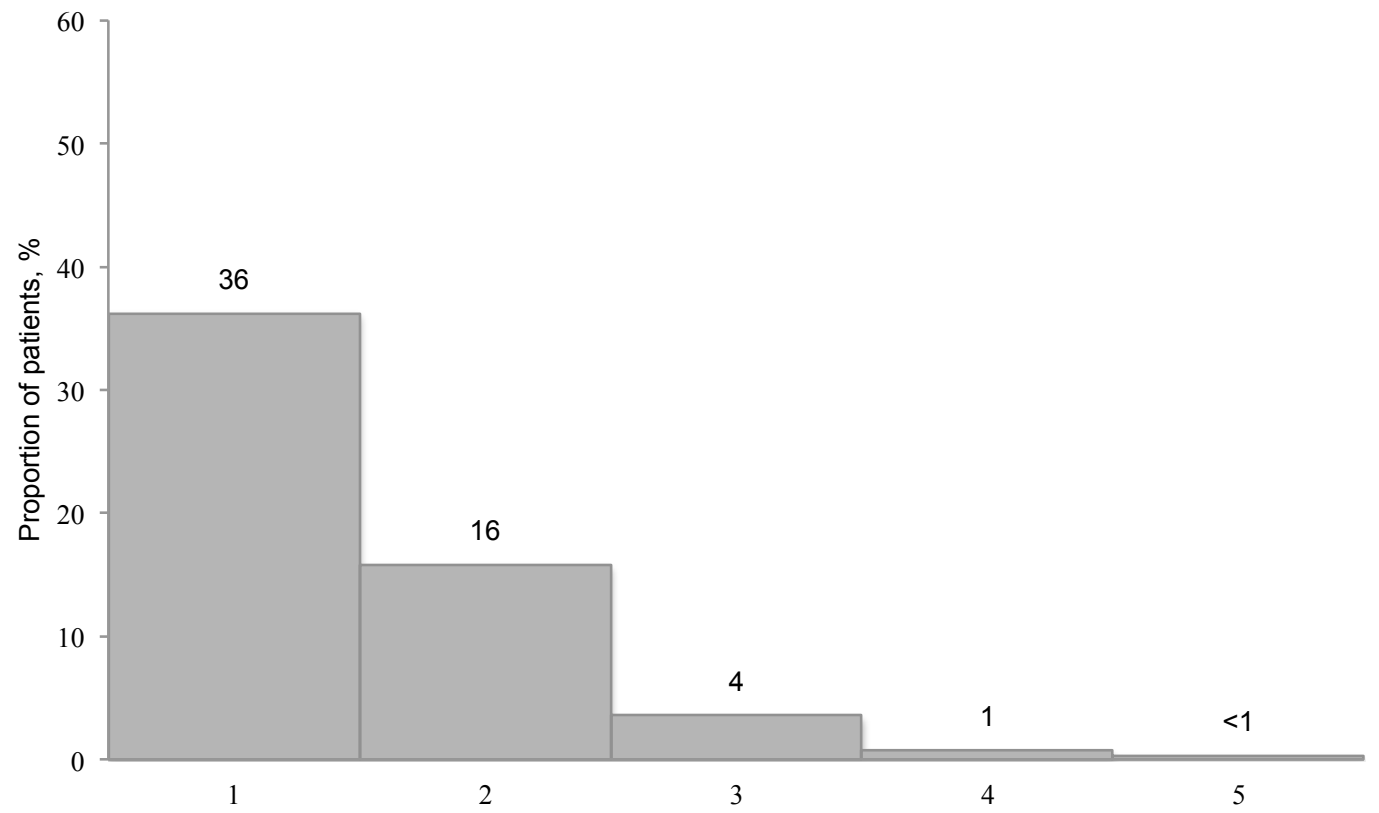

Number of PIMs per patient 


\section{Figure 3}

OR $(95 \% \mathrm{CI})$

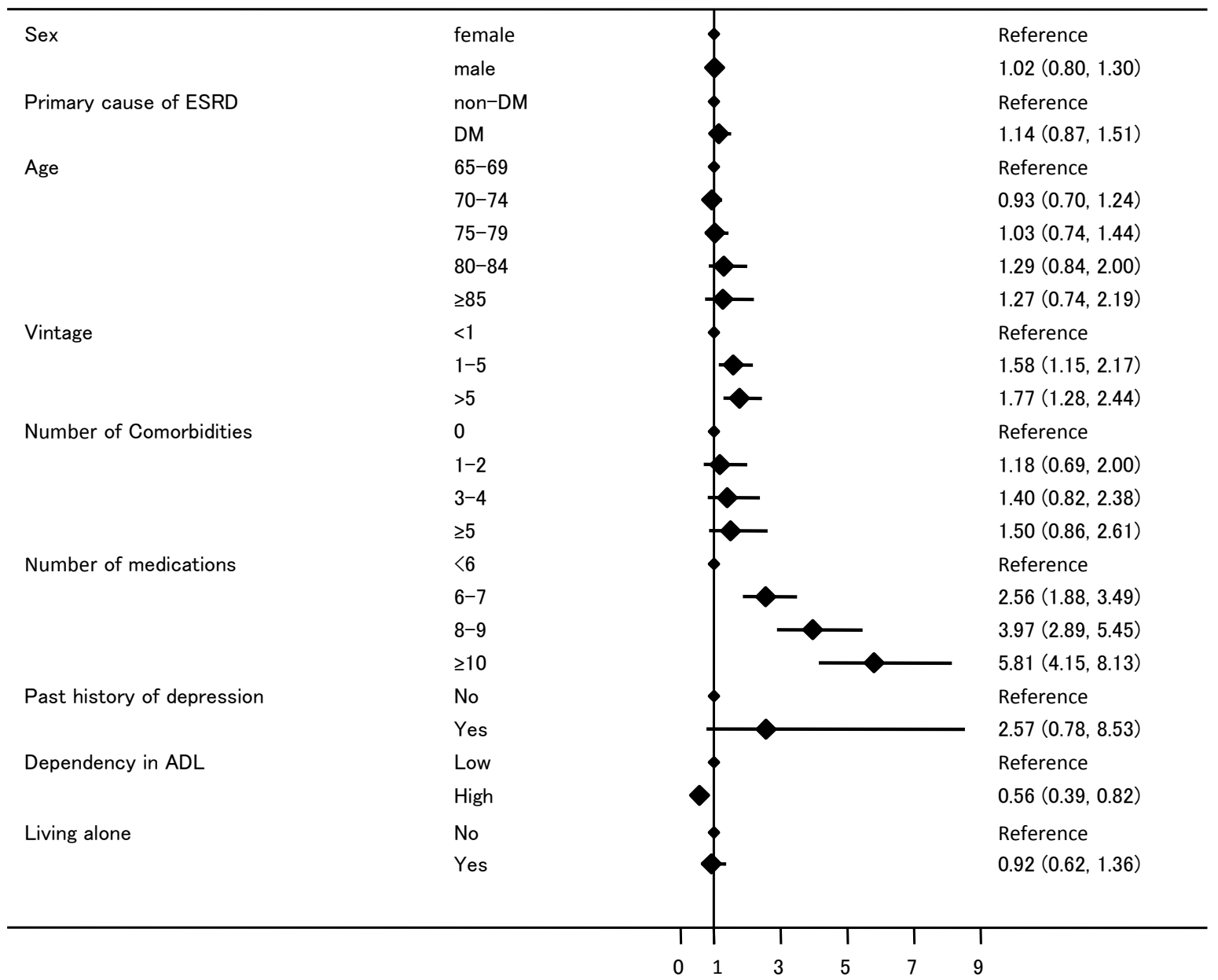


Figure 4

OR $(95 \% \mathrm{CI})$

\begin{tabular}{|c|c|c|}
\hline \multirow[t]{2}{*}{ Number of HD stations } & $<30$ & Reference \\
\hline & $\geq 30$ & $0.79(0.56,1.12)$ \\
\hline \multirow[t]{2}{*}{ Teaching hospital } & No & Reference \\
\hline & Yes & $0.56(0.38,0.85)$ \\
\hline \multirow[t]{2}{*}{ Multidisciplinary rounds conducted } & No & Reference \\
\hline & Yes & $0.66(0.48,0.92)$ \\
\hline \multirow{2}{*}{$\begin{array}{l}\text { Hemodialysis regimen able to be changed by more } \\
\text { than one physician }\end{array}$} & No & Reference \\
\hline & Yes & $1.66(1.13,2.46)$ \\
\hline & & 3 \\
\hline
\end{tabular}


This is a pre-copyedited, author-produced PDF of an article accepted for publication in Nephrology Dialysis Transplantation following peer review. The version of record "Prescription of potentially inappropriate medications to elderly hemodialysis patients: prevalence and predictors" is available online at: http://ndt.oxfordjournals.org/content/early/2014/04/26/ndt.gfu 070.short. 